\title{
ON THE NUMBER OF BRANCHES OF A PLANE CURVE GERM
}

\author{
By Takuo Fukuda, Kenji Aoki and Wei-Zhi Sun
}

\section{Introduction}

The topology of the zero locus $f^{-1}(0)$ of a smooth map-germ $f:\left(R^{n}, 0\right) \rightarrow\left(R^{p}, 0\right)$ is one of the most important and interesting problems in singularity theory. Moreover it determines the homological type of $f$ (see $\S 3$ ). In this note we study the simplest case of smooth functions of two variables.

Let $f:\left(R^{2}, 0\right) \rightarrow(R, 0)$ be a $C^{\infty}$ function with an isolated critical point 0 . For a small positive number $\varepsilon>0$, set

$$
\begin{aligned}
& B_{\varepsilon}=\left\{(x, y): x^{2}+y^{2} \leqq \varepsilon\right\} \\
& S_{\varepsilon}^{1}=\left\{(x, y): x^{2}+y^{2}=\varepsilon\right\} .
\end{aligned}
$$

The connected components of the set $B_{\varepsilon} \cap f^{-1}(0)-\{0\}$ are called branches of $f^{-1}(0)$. We adopt this definition for our convenience, though it might differ from the usual one, (if it exists.) The number of branches of $f^{-1}(0)$ coincides with the number of the connected components of $S_{\varepsilon}^{1} \cap f^{-1}(0)$, and it determines not only the topological type of $f^{-1}(0)$ but also the topological type of $f$. In this note we give an algebraic formula for the number of branches of a plane curve germ $f^{-1}(0)$.

Given a function $f:\left(R^{2}, 0\right) \rightarrow(R, 0)$, set

$$
J_{f}=\left|\begin{array}{cc}
\partial f / \partial x & \partial f / \partial y \\
x & y
\end{array}\right|=\frac{1}{2} \operatorname{Jacobian}\left(f, x^{2}+y^{2}\right) .
$$

Then our first result is

THEOREM 1. Let $f:\left(R^{2}, 0\right) \rightarrow(R, 0)$ be a function germ with an isolated critical point 0 such that 0 is also an isolated critical point of $J_{f}$. Then we have the number of branches of $f^{-1}(0)=2\left|\operatorname{deg}\left(f, J_{f}\right)\right|$, where $\left|\operatorname{deg}\left(f, J_{f}\right)\right|$ denotes the absolute value of the topological degree of the mapping $\left(f, J_{f}\right) /\left\|\left(f, J_{f}\right)\right\|: S_{\varepsilon}^{1} \rightarrow S^{1}$.

Thanks to Eisenbud and Levine's theorem ([1]), we can calculate $\left|\operatorname{deg}\left(f, J_{f}\right)\right|$ in terms of $\mathrm{J}$. Mather's local algebra $Q\left(f, J_{f}\right)$ associated to the map germ $\left(f, J_{f}\right):\left(R^{2}, 0\right) \rightarrow\left(R^{2}, 0\right):$

Received September 4, 1985 


$$
\left|\operatorname{deg}\left(f, J_{f}\right)\right|=\operatorname{dim}_{R} Q\left(f, J_{f}\right)-2 \operatorname{dim}_{R} I\left(f, J_{f}\right),
$$

where $I=I\left(f, J_{f}\right)$ is an ideal of $Q\left(f, J_{f}\right)$ which is maximal with respect to the property $I^{2}=0$. Thus we have

THEOREM 2. If $f:\left(R^{2}, 0\right) \rightarrow(R, 0)$ is a $C^{\infty}$ function germ with an isolated critical point such that $\operatorname{dim}_{R} Q\left(f, J_{f}\right)<\infty$, then the number of branches of $f^{-1}(0)=2\left\{\operatorname{dim}_{R} Q\left(f, J_{f}\right)-2 \operatorname{dim}_{R} I\left(f, J_{f}\right)\right\}$.

Our motivation of this study was a desire to discover a generalization of Eisenbud and Levine's beautiful theorem. If one wants to define degree of a map germ $f:\left(R^{n}, 0\right) \rightarrow\left(R^{p}, 0\right)$ for a general pair $(n, p)$ of dimensions, there seems to be two ways, the homological one and the homotopical one. In $\$ 3$ we discuss the homological version and the importance of the number of connected components of $f^{-1}(0) \cap S_{\varepsilon}^{n-1}$ is emphasized.

It should be noted that C. T. C. Wall ([3]) proved implicitly that for a $C^{\infty}$ function germ $f:\left(R^{n}, 0\right) \rightarrow(R, 0)$ with an isolated critical point 0 , we have

$$
\chi\left(S_{\varepsilon}^{n-1} \cap f^{-1}(0)\right)=-\operatorname{deg}(d f)+(-1)^{n-1} \operatorname{deg}(d f)+2-\chi\left(S^{n-1}\right),
$$

where $d f=\left(\partial f / \partial x_{1}, \cdots, \partial f / \partial x_{n}\right):\left(R^{n}, 0\right) \rightarrow\left(R^{n}, 0\right)$ and $\chi$ denotes the Euler characteristic.

In the case where $n=2$, the Euler characteristic of $S_{\varepsilon}^{1} \cap f^{-1}(0)$ is the number of branches of $f^{-1}(0)$ in our sense. Therefore ours is not the first formula for the number of branches of plane curve germs. Moreover it is often easier to calculate the degree of $(\partial f / \partial x, \partial f / \partial y)$ than the degree of $\left(f, J_{f}\right)$. However our result possibly has the merit of generalizing in another direction, that is to a formula for the number of branches of $f^{-1}(0)$ for a map germ $f:\left(R^{n}, 0\right) \rightarrow$ $\left(R^{n-1}, 0\right)$; from our experiments on examples, the following conjecture seems likely to be true.

CONJECTURE. Let $f=\left(f_{1}, \cdots, f_{n-1}\right):\left(R^{n}, 0\right) \rightarrow\left(R^{n-1}, 0\right)$ be a map germ with a generic condition. Set

$$
\begin{aligned}
J_{f}= & \text { the jacobian determinant of the map germ } \\
& \left(f_{1}, \cdots, f_{n-1}, x_{1}^{2}+\cdots x_{n}^{2}\right):\left(R^{n}, 0\right) \rightarrow\left(R^{n}, 0\right) .
\end{aligned}
$$

Then the number of branches of $f^{-1}(0)$ is equal to twice the absolute value of the degree of $\left(f, J_{f}\right):\left(R^{n}, 0\right) \rightarrow\left(R^{n}, 0\right)$.

\section{Proof of theorem 1 .}

Theorem 1 follows from the following two lemmas.

LEMMA 2.1. Let $f, g:\left(R^{2}, 0\right) \rightarrow(R, 0)$ be $C^{\infty}$ function germs with isolated critical points such that $f^{-1}(0) \cap g^{-1}(0)=\{0\}$. Suppose that for any two adjacent 
branches of $f^{-1}(0), g^{-1}(0)$ has an odd number of branches between them. Then we have the number of branches of $f^{-1}(0)=2|\operatorname{deg}(f, g)|$.

LEMMA 2.2. Let $f:\left(R^{2}, 0\right) \rightarrow(R, 0)$ be a $C^{\infty}$ function with an isolated critical point 0. Suppose that

$$
J=y \cdot \partial f / \partial x-x \cdot \partial f / \partial y
$$

has 0 as an isolated critical point. Then $J^{-1}(0)$ has an odd number of branches between any pair of adjacent branches of $f^{-1}(0)$.

Proof of Lemma 2.1. Take any branch, say $a_{1}$, of $f^{-1}(0)$. Let $a_{2}$ be the one next to $a_{1}$ anticlockwisely and $a_{3}$ the one next to $a_{2}$. From the hypothese, there are an odd number of branches of $g^{-1}(0)$, say $b_{1}, \cdots, b_{2 k+1}$, between $a_{1}$ and $a_{2}$, and also an odd number of branches of $g^{-1}(0)$, say $c_{1}, \cdots, c_{2 m+1}$, between $a_{2}$ and $a_{3}$. For simplicity, we give a proof for the case where $k=1$ and $m=0$. The proof for the general case is similar. Let $D_{1}, D_{2}, \cdots, D_{6}$ be the regions between the branches of $f^{-1}(0)$ and $g^{-1}(0)$. (See Figure 2.1.)

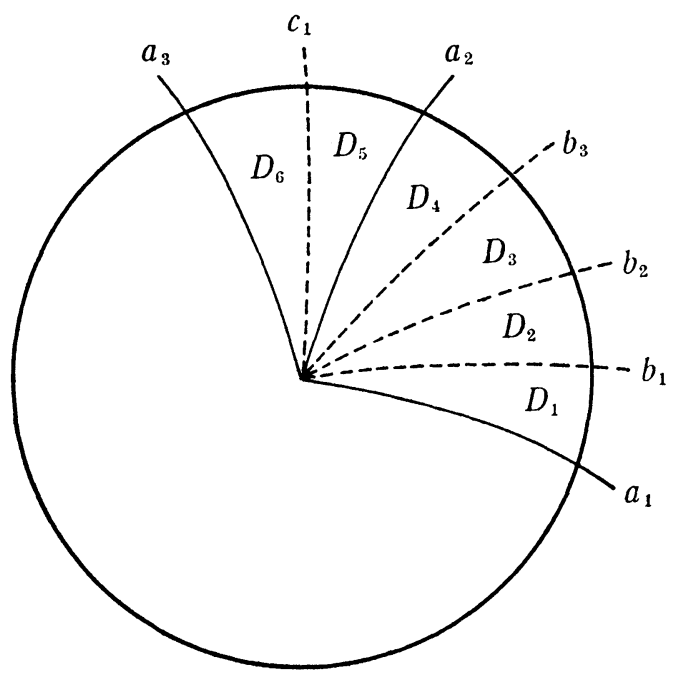

(Figure 2.1)

Let $\varepsilon$ be a sufficiently small positive number. Since 0 is an isolated critical point of $f$, the function $f_{\varepsilon}(\theta)=f(\varepsilon \cos \theta, \varepsilon \sin \theta)$ changes the sign of its value when the path $\varepsilon e^{i \theta}=(\varepsilon \cos \theta, \varepsilon \sin \theta)$ passes through a branch of $f^{-1}(0)$. Ditto for $g(\varepsilon \cos \theta, \varepsilon \sin \theta)$. Without losing generality, we may suppose $f>0$ and $g>0$ in $D_{1}$. Then we have the following table of the signs of values of $f$ and $g$. 


\begin{tabular}{|l|c|c|c|c|c|c|c|c|c|c|c|c|c|}
\hline$\varepsilon e^{i \theta}$ & $a_{1}$ & $D_{1}$ & $b_{1}$ & $D_{2}$ & $b_{2}$ & $D_{3}$ & $b_{3}$ & $D_{4}$ & $a_{2}$ & $D_{5}$ & $c_{1}$ & $D_{6}$ & $a_{3}$ \\
\hline$X=f$ & 0 & + & + & + & + & + & + & + & 0 & - & - & - & 0 \\
\hline$Y=g$ & + & + & 0 & - & 0 & + & 0 & - & - & - & 0 & + & + \\
\hline
\end{tabular}

From the table, we have the following figure which shows the image of the $\operatorname{map}(f(\varepsilon \cos \theta, \varepsilon \sin \theta), g(\varepsilon \cos \theta, \varepsilon \sin \theta))$.

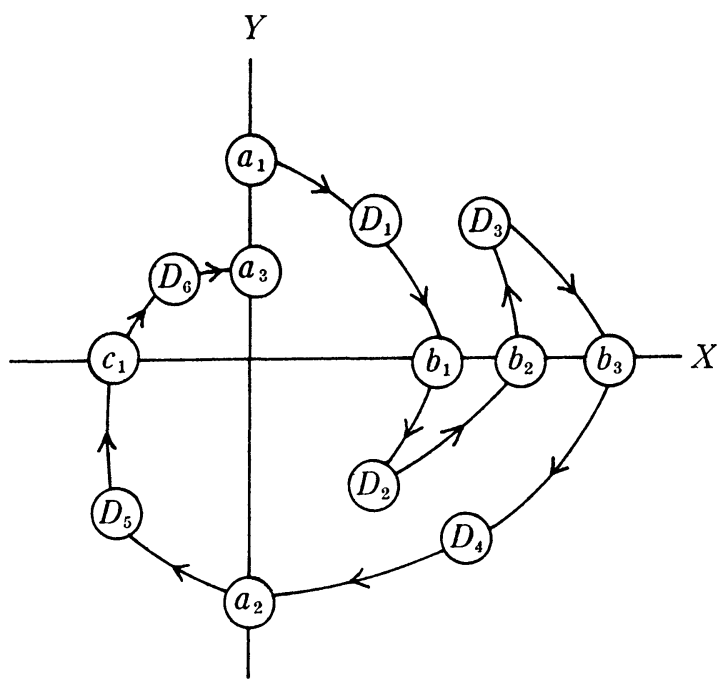

(Figure 2.2)

From the above figure, we see that as the path $(\varepsilon \cos \theta, \varepsilon \sin \theta)$ goes from $a_{1}$ to $a_{3}$ via $a_{2}$, the degree of $(f, g)$ increases by -1 . In the above argument it does not matter how many branches $g^{-1}(0)$ has, so long as it has an odd number of branches between each two adjacent branches of $f^{-1}(0)$.

Q. E. D. of Lemma 2.1 .

Proof of Lemma 2.2. Now we use the polar coordinates $(r, \theta)$ :

$$
x=r \cos \theta, \quad y=r \sin \theta .
$$

For a small positive number $r$, we set

$$
f_{r}(\theta)=f(r \cos \theta, r \sin \theta) .
$$

Then we have 


$$
\begin{aligned}
d f_{r} / d \theta & =\partial f / \partial x \cdot d(r \cos \theta) / d \theta+\partial f / \partial y \cdot d(r \sin \theta) / d \theta \\
& =-r \sin \theta \cdot \partial f / \partial x+r \cos \theta \cdot \partial f / \partial y \\
& =-y \cdot \partial f / \partial x+x \cdot \partial f / \partial y=J_{f}
\end{aligned}
$$

Since 0 is a common isolated critical point of $f$ and $J_{f}, f_{r}(\theta)$ is a Morse function of the variable $\theta$ and $f_{r}(\theta)$ changes its $\operatorname{sign}$ when the path $(r \cos \theta$, $r \sin \theta$ ) passes through a branch of $f^{-1}(0)$. Thus we have

$$
J_{f}=-d f_{r} / d \theta \neq 0 \quad \text { on } \quad f^{-1}(0) .
$$

Let

$$
a_{1}=\left(r \cos \theta_{1}, r \sin \theta_{1}\right) \text { and } a_{2}=\left(r \cos \theta_{2}, r \sin \theta_{2}\right)
$$

be any two points of $f^{-1}(0) \cap\left\{x^{2}+y^{2}=r^{2}\right\}$ which are next to each other.

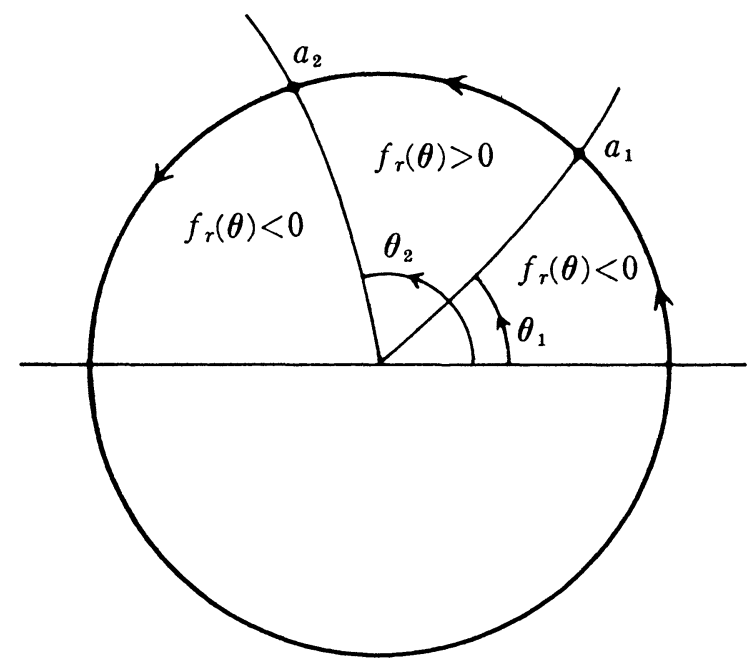

(Figure 2.3)

Since 0 is an isolated critical point of $f$, the sign of value of $f_{r}(\theta)$ changes at $\theta_{1}$ and $\theta_{2}$ and it does not change between them. We may suppose without losing generality that $f_{r}(\theta)>0$ if $\theta_{1}<\theta<\theta_{2}$. Thus we have

$$
d f_{r} / d \boldsymbol{\theta}\left(\boldsymbol{\theta}_{1}\right)>0 \text { and } d f_{r} / d \theta\left(\theta_{2}\right)<0 .
$$

Since $f_{r}(\theta)$ is a Morse function, it has an odd number of critical points between $\theta_{1}$ and $\theta_{2}$. (See the figure below.) 


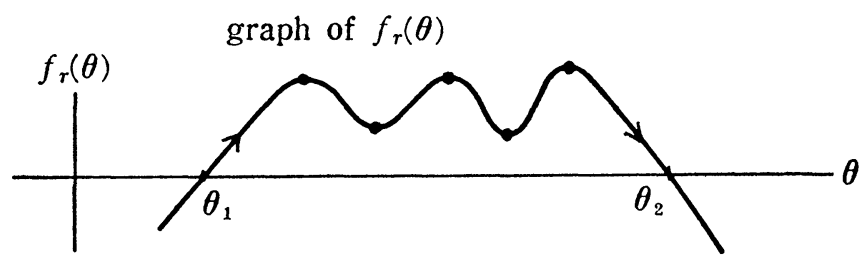

(Figure 2.4)

Since

$$
\text { \{critical points of } \begin{aligned}
\left.f_{r}(\theta)\right\} & =\left\{\theta \mid d f_{r} / d \theta=0\right\} \\
& =\left\{J=0, x^{2}+y^{2}=r^{2}\right\},
\end{aligned}
$$

the number of branches of $J^{-1}(0)$ between a pair of adjacent branches of $f^{-1}(0)$ is odd.

Q. E. D. of Lemma 2.2 .

\section{Homological behaviors of a map germ}

In this section, we attempt to generalize the notion of degree of map germs : $\left(R^{n}, 0\right) \rightarrow\left(R^{n}, 0\right)$ to the case of map germ: $\left(R^{n}, 0\right) \rightarrow\left(R^{p}, 0\right)$ with $n>p$ from a homological view point. We propose here to define the absolute value $|\operatorname{deg} f|$ of the homological degree of $f$ to be the number of connected components of $f^{-1}(0) \cap S_{\varepsilon}^{n-1}$.

In the case where $n=p$, a map germ $f:\left(R^{n}, 0\right) \rightarrow\left(R^{n}, 0\right)$ such that $f^{-1}(0)=\{0\}$ induces a homomorphism

$$
f_{*}: H_{n-1}\left(R^{n}-\{0\} ; Z\right) \cong Z \rightarrow H_{n-1}\left(R^{n}-\{0\} ; Z\right) \cong Z .
$$

The induced homomorphism $f_{*}$ may be regarded as multiplication in $Z$ by some integer $d ; f_{*}(a)=d . a$. The integer $d$ thus associated to $f_{*}$ is the degree of $f:\left(R^{n}, 0\right) \rightarrow\left(R^{n}, 0\right)$ (see [1]). So, if we want to generalize it to the general case, first we should observe the homology groups of $R^{n}-f^{-1}(0)$ and $R^{p}-\{0\}$ and the homomorphisms $f_{*}$ between them.

Let $f:\left(R^{n}, 0\right) \rightarrow\left(R^{p}, 0\right)$ be a $C^{\infty}$ map germ such that 0 is an isolated singular point of $f^{-1}(0)$. Let $D_{\varepsilon}^{n}$ and $S_{\varepsilon}^{n-1}$ be the closed $n$-disk and the $(n-1)$-sphere in $R^{n}$ centered at 0 with radius $\varepsilon$ respectively. Then we must observe the homomorphisms

$$
f_{*}: H_{\imath}\left(D_{\varepsilon}^{n}-f^{-1}(0): Z\right) \rightarrow H_{i}\left(R^{p}-\{0\}: Z\right), \quad \imath=0,1, \cdots, p-1 .
$$

Here we choose a representative mapping of $f$ and denote it also by the same notation $f$.

Since

$$
\begin{array}{cl}
H_{i}\left(R^{p}-\{0\} ; Z\right) \cong Z & \text { for } \imath=0 \text { and } \imath=p-1 \\
Z \oplus Z & \text { for } i=0 \text { and } p=1 \\
0 & \text { otherwise, }
\end{array}
$$


the homomorphisms

$$
f_{*}: H_{i}\left(D_{\varepsilon}^{n}-f^{-1}(0) ; Z\right) \rightarrow H_{i}\left(R^{p}-\{0\} ; Z\right)
$$

are 0 -homomorphisms for $i \neq 0, p-1$. Hence it suffices to observe

and

$$
f_{*}: H_{0}\left(D_{\varepsilon}^{n}-f^{-1}(0) ; Z\right) \rightarrow H_{0}\left(R^{p}-\{0\} ; Z\right)
$$

$$
f_{*}: H_{p-1}\left(D_{\varepsilon}^{n}-f^{-1}(0) ; Z\right) \rightarrow H_{p-1}\left(R^{p}-\{0\} ; Z\right) \quad \text { for } \quad p>1 .
$$

Now, using the Alexander-Pontrjagin duality and the Poincaré duality, we can express homology groups $H_{i}\left(D_{\varepsilon}^{n}-f^{-1}(0) ; Z\right)$ in terms of $H_{j}\left(S_{\varepsilon}^{n-1} \cap f^{-1}(0) ; Z\right)$, in particular we have

2) if $n-p-1>0$ and $p>1$, then

$$
\begin{aligned}
H_{p-1}\left(D_{\varepsilon}^{n}-f^{-1}(0) ; Z\right) & \cong H_{p-1}\left(S_{\varepsilon}^{n-1}-f^{-1}(0) ; Z\right) \\
& \cong H_{0}\left(S_{\varepsilon}^{n} \cap f^{-1}(0) ; Z\right)
\end{aligned}
$$

3) if $n-p-1=0$ and $p>1$, then

$$
\begin{aligned}
H_{p-1}\left(D_{\varepsilon}^{n}-f^{-1}(0) ; Z\right) \oplus Z & \cong H_{p-1}\left(S_{\varepsilon}^{n-1}-f^{-1}(0) ; Z\right) \oplus Z \\
& \cong H_{0}\left(S_{\varepsilon}^{n-1} \cap f^{-1}(0) ; Z\right)
\end{aligned}
$$

Thus the homology groups we want to know turn out to be

$$
H_{0}\left(S_{\varepsilon}^{n-1} \cap f^{-1}(0) ; Z\right) \text { and } H_{0}\left(S_{\varepsilon}^{n-1}-f^{-1}(0) ; Z\right)
$$

which are completely determined by the number of connected components of $S_{\varepsilon}^{n-1} \cap f^{-1}(0)$ and $S_{\varepsilon}^{n-1}-f^{-1}(0)$ respectively.

Now let us observe

$$
f_{*}: H_{0}\left(D_{\varepsilon}^{n}-f^{-1}(0) ; Z\right) \rightarrow H_{0}\left(R^{p}-\{0\} ; Z\right) .
$$

This homomorphism is rather trivial in the following sense:

a) If $p>1$, then $D_{\varepsilon}^{n}-f^{-1}(0)$ is connected and hence

$$
f_{*} ; H_{0}\left(D_{\varepsilon}^{n}-f^{-1}(0) ; Z\right) \cong Z \rightarrow H_{0}\left(R^{p}-\{0\} ; Z\right) \cong Z
$$

may be regarded as the identity map of $Z$.

b) If $p=1$, then

$$
f_{*}: H_{0}\left(D_{\varepsilon}^{n}-f^{-1}(0) ; Z\right) \rightarrow H_{0}(R-\{0\} ; Z) \cong Z \oplus Z
$$

is completely determined by the number of connected components of $D_{\varepsilon}^{n} \quad f^{-1}(0)$ and the sign of values of $f$ on each component.

Now let us observe 


$$
f_{*}: H_{p-1}\left(D_{\varepsilon}^{n}-f^{-1}(0) ; Z\right) \rightarrow H_{p-1}\left(R^{p}-\{0\} ; Z\right) \cong Z .
$$

Let $K_{\imath}, i=1,2, m, \cdots, k$, be the connected components of $S_{\varepsilon}^{n-1} \cap f^{-1}(0)$. Since 0 is an isolated singular point of $f^{-1}(0)$, the restricted mapping $f \mid S_{\varepsilon}^{n-1}: S_{\varepsilon}^{n-1} \rightarrow R^{p}$ is submersive in a neighbourhood of $K_{\imath}$. Hence $K_{\imath}$ has a tubular neighbourhood $T_{\imath}$ in $S_{\varepsilon}^{n-1}$ which is diffeomorphic to $K_{\imath} \times D_{\delta}^{p}$ for a small positive number $\delta$. Identifying $T_{\imath}$ with $K_{\imath} \times D_{\delta}^{p}$, the restricted mapping $f \mid K_{\imath} \times D_{\delta}^{p}: K_{\imath} \times D_{\delta}^{p} \rightarrow D_{\delta}^{p} \subset R^{p}$ becomes the canonical projection.

Thus and from the above duality isomorphisms 2) and 3 ), letting $q_{2}$ be a point of $K_{\imath}$, we see that the homology class

$$
\begin{aligned}
{\left[\left\{q_{i}\right\} \times \partial D_{\delta}^{p}\right] } & \in H_{p-1}\left(S_{\varepsilon}^{n-1}-f^{-1}(0) ; Z\right) \\
& =H_{p-1}\left(D_{\varepsilon}^{n}-f^{-1}(0) ; Z\right)
\end{aligned}
$$

are the generators of $H_{p-1}\left(D_{\varepsilon}^{n}-f^{-1}(0) ; Z\right)$ and also that

$$
f_{*}\left(\left[\left\{q_{i}\right\} \times \partial D_{\delta}^{p}\right]\right)= \pm\left[\partial D_{\delta}^{p}\right]= \pm 1 \in H_{p-1}\left(R^{p}-\{0\} ; Z\right) .
$$

The sign in the above equality depends only on the choice of orientation of $\left\{q_{i}\right\} \times \partial D^{p}$. Thus we have

Proposition. The homological behavior of $f$ is completely determined by the homology groups of $S_{\varepsilon}^{n-1} \cap f^{-1}(0)$ and the orientations. In particular the homomorphism

$$
f_{*}: H_{p-1}\left(D_{\varepsilon}^{n}-f^{-1}(0) ; Z\right) \rightarrow H_{p-1}\left(R^{p}-\{0\} ; Z\right) \quad p>1
$$

is completely determined by the number of connected components of $S_{\varepsilon}^{n-1} \cap f^{-1}(0)$ and the orientations.

As a conclusion of this section, we emphasize the importance of the number of connected components of $S_{\varepsilon}^{n-1} \cap f^{-1}(0)$ which can be regarded as the absolute value $|\operatorname{deg} f|$ of the degree of $f:\left(R^{n}, 0\right) \rightarrow\left(R^{p}, 0\right)$. In particular in the case where $p=n-1$, the homological behaviour of $f$ is completely determined by the number of connected components of $S_{\varepsilon}^{n-1} \cap f^{-1}(0)$ which is equal to the number of branches of $f^{-1}(0)$. Thus we are interested in the conjecture given at the end of $\S 1$.

\section{REFERENCES}

[1] D. Eisenbud And H.I. Levine, An algebraic formula for the degree of a $C^{\infty}$ map-germ. Ann. Math. 106 (1977), 19-38.

[2] J. MILnOR, Topology from the differential view point. The University Press of Virginia, Charlottesville, 1965.

[3] C.T.C. WaLl, Topological invariance of the Milnor number mod 2. Topology 22 (1983), 345-350. 
Department of Mathematics

Tokyo Institute of Technology

OH-OKay ama MEguro-Ku TOKYo, JaPAN

Department of Information Sciences

Nihon Kogakuin Technical College

Nishikamata OHTa-kU TOKYo, JaPan

Department of Mathematics

Dong Bei Normal University

ChANG $C_{\text {HUN, }}$ ChINA 